J Neurol Neurosurg Psychiatry 2005;76(Suppl II):ii8-ii12. doi: 10.1136/jnnp.2005.068486

$\Lambda$ lthough the electroencephalogram (EEG) is a reliable test to assess cerebral function, its value in diagnosis and evaluation of neurological conditions apart from epilepsy has been largely superceded in recent years by other investigations with greater specificity and sensitivity. Is EEG still worthwhile, and in which cases can it provide information that affects management? Broadly speaking, EEG is most important in patients with impaired consciousness or altered mental state (table 1 ):

- Where seizures or non-convulsive status epilepticus (NCSE) may be a contributing factor

- To demonstrate functional disturbance when cerebral dysfunction is evident and structural imaging is normal

- To detect focal or lateralised abnormalities which could suggest a structural basis for an encephalopathy

- To identify diagnostic EEG patterns in appropriate clinical settings, such as sporadic Creutzfeldt-Jakob disease (CJD).

\title{
ENCEPHALOPATHIES
}

EEG changes in encephalopathies are similar, whether the cause is septic, metabolic, toxic, or structural. There is a progressive increase in slow wave activities, the degree of which parallels the severity of brain dysfunction. In mild encephalopathic states, slowing of normal alpha $(\alpha)$ rhythms occurs, and with more severe encephalopathy, the appearance of theta $(\theta)$ and continuous or non-continuous delta $(\delta)$ activities. A variety of additional EEG patterns can be seen, such as frontal intermittent rhythmic delta (FIRDA), periodic lateralised or bilateral epileptiform discharges (PEDs, BIPEDs), and triphasic waves. None of these patterns is specific to a particular pathophysiological process or diagnosis, but PEDs are most likely to occur in acute or subacute focal destructive pathologies or focal epileptogenic lesions; triphasic waves are typically found in metabolic encephalopathies; and some patients with mesial fronto-parietal lesions or third ventricle tumours show FIRDA in their EEG.

\section{Metabolic encephalopathies}

A patient with acute change in awareness whose EEG shows triphasic waves and diffuse slow activity will usually have a metabolic encephalopathy. Triphasic waves (TW) consist of moderate to high amplitude complexes with three (but sometimes two or four) negative-positive-negative phases, usually occurring in runs at 1.5-3 per second. They have been considered as specific to severe hepatic encephalopathy, but TW are seen in encephalopathies associated with renal failure or electrolyte imbalance, as well as anoxia and intoxications (such as lithium, metrizamide, and levodopa). The rate of change rather than the absolute level of a given metabolite, toxin or electrolyte is more important in determining the degree of EEG abnormality. Thus, EEG changes are usually more severe in uraemic encephalopathy if there is acute deterioration of renal function, with broad correlation between degree of EEG slowing and increase in serum creatinine. Spike wave epileptiform discharges (and seizures) may occur in any severe encephalopathy, but are said to be more common than in uraemic encephalopathy and in insulin coma.

In hypoglycaemia there may be generalised slow activity or focal/lateralised $\delta$ rhythms, and these may be associated with focal neurological deficits.

EEG can be useful for early detection of dialysis dementia or encephalopathy (occurring in about $1 \%$ of dialysis patients), as abnormalities can precede clinical symptoms by several months in this disorder.

Correspondence to: Dr Shelagh Smith, Department of Clinical Neurophysiology, The National Hospital, Queen Square, London WCIN 3BG, UK; shelaghs@epilepsynse. org.uk

\section{Toxic encephalopathies}

Most drugs or toxins have diffuse effects on the EEG. Some agents, particularly benzodiazepines and barbiturates, induce fast or beta $(\beta)$ rhythms, and the EEG may be a useful pointer to drug intoxication when this is clinically unsuspected. Generalised slow activity can alert to hyperammonaemia in a confused or obtunded patient who has epilepsy treated with sodium 
Table 1 Strengths and weaknesses of the EEG

EEG is useful for: Identifying seizures or NCSE

Distinguishing organic disorders

from psychiatric/functional illness

Predicting outcome of some types Early detec

of coma

Identifying reversible causes of

cognitive impairment

NCSE, non-convulsive status epilepticus.

valproate. Clozapine is quite commonly associated with EEG change, often pronounced, and manifest by both generalised slow activity and spike wave discharge in non-epileptic patients. Induction of clinical seizures and EEG abnormalities are more likely at high dosages or with rapid dose escalation.

\section{Endocrine disorders}

EEG changes parallel the severity of hormonal disturbance, but it may be difficult to distinguish primary endocrine from secondary electrolyte effect. Hashimoto's thyroiditis has heterogeneous clinical and electrographic manifestations; the EEG can show generalised or frontal slow activity, triphasic waves, periodic sharp waves, and lateralised temporal slowing.

\section{Anoxic encephalopathy}

Most studies have involved patients with ischaemic/anoxic brain damage following prolonged cardiorespiratory arrest, and EEG grading systems have been proposed for prognostic purposes. Some patterns-burst suppression and isoelectric EEG-are particularly associated with poor outcome (death, vegetative state), and PEDs or BIPEDs are also adverse electrographic findings. However, great caution must be exercised when judging prognosis based on a single EEG recording, and it is essential to consider other factors such as systemic complications, electrolyte imbalance, core temperature, and use of sedative medication that may reversibly affect cerebral function and the EEG. EEG variability, either spontaneous or in reaction to external stimuli, is an important feature, and reactivity to a range of stimulation procedures (auditory, pain) should be assessed during the EEG recording.

\section{When should EEG be performed in the intensive care patient?}

- To detect non-convulsive or clinically subtle seizures. Most cases of status on the intensive care unit (ICU) are nonconvulsive or difficult to identify on clinical grounds alone. NCSE should be anticipated if there is a prior history of epilepsy; in patients with CNS infection, recent neurosurgical procedures, head injury or stroke; as a sequela of convulsive status epilepticus; and following prolonged cardiorespiratory arrest. Up to $10 \%$ of patients with unexplained coma have NCSE, based on electrographic studies. Occurrence of NCSE and delay in recognition/treatment are associated with poorer outcome independent of aetiology of status and age.

- To characterise paroxysmal clinical events that might be seizures, including grimacing, chewing, or nystagmoid eye movements; abrupt and otherwise unexplained changes in pulse, blood pressure or respiratory pattern; or abrupt deterioration in conscious level. Ideally, video and EEG should be recorded concurrently.
- To distinguish coma from diminished responsiveness due to other causes (psychiatric, sedation, neuromuscular, cortical de-efferentation/locked in syndrome).

The use of EEG for detection of cerebral ischaemia at a reversible stage, such as following subarachnoid haemorrhage, is currently a research tool. Systems that utilise EEG measures to monitor the level of sedation or anaesthesia have not been widely accepted for either perioperative or intensive care use. Neurophysiological criteria-preferably a combination of EEG, somatosensory N20, and mid-latency auditory evoked potentials, central motor conduction time-have been shown to predict outcome of coma, albeit in studies mostly limited to patients with anoxic-ischaemic or traumatic brain damage. That such techniques are not in widespread use is partly a reflection of limited service availability, and perhaps the technical challenges of continuous neurophysiological monitoring in particular.

EEG no longer has a role in determination of brain death, but demonstration of an isoelectric and wholly unreactive EEG, in addition to other assessment of the patient, may sometimes help relatives accept that ongoing treatment is futile.

\section{NEUROLOGICAL INFECTIONS}

EEG is always abnormal in acute encephalitis, and demonstration of slow activity in a confused/obtunded patient with pyrexia helps to confirm the diagnosis. Electrographic abnormalities will be non-specific as to cause, although focal or lateralised changes can raise suspicion of encephalitis caused by herpes simplex infection (HSE). These include focal $\delta$ activity and PEDs, typically over the temporal lobes (fig 1), with either unilateral or bilateral involvement. PEDs were considered to be the EEG hallmark of HSE, but they are also seen in other acute encephalitides, brain tumours, and acute strokes. Moreover, it may take several days for the PEDs to appear in HSE, the discharges can be transient, and there is some evidence that prompt treatment with antiviral therapy reduces the likelihood that PEDs will occur. Thus, the role of EEG in diagnosis of HSE has been overtaken by specific immunological and viral detection tests in CSF and neuroimaging. Any patient now with suspected encephalitis will be treated with acyclovir from the time of presentation in the accident and emergency department, without delay introduced by waiting for an EEG. EEG findings in HSE may give prognostic information, as cases with bilateral epileptiform discharges tend to have poor long term

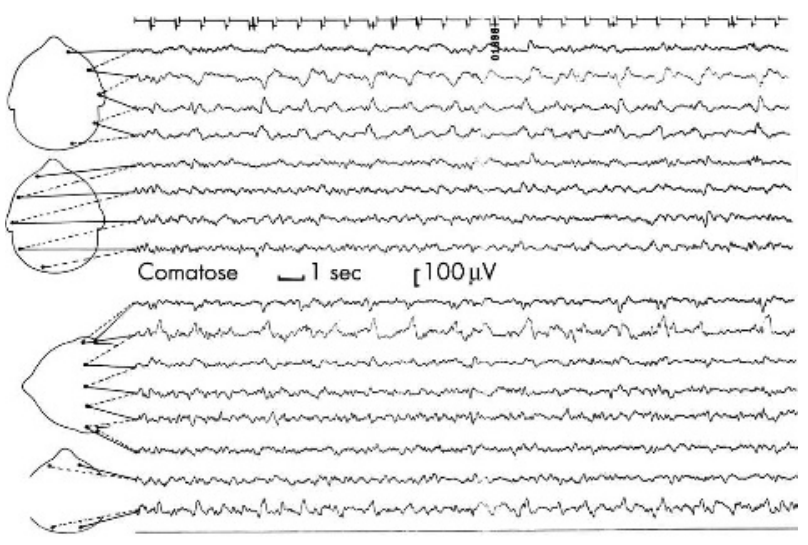

Figure 1 Herpes simplex encephalitis: left sided slow activity and repetitive periodic epileptiform discharges (PEDs). 


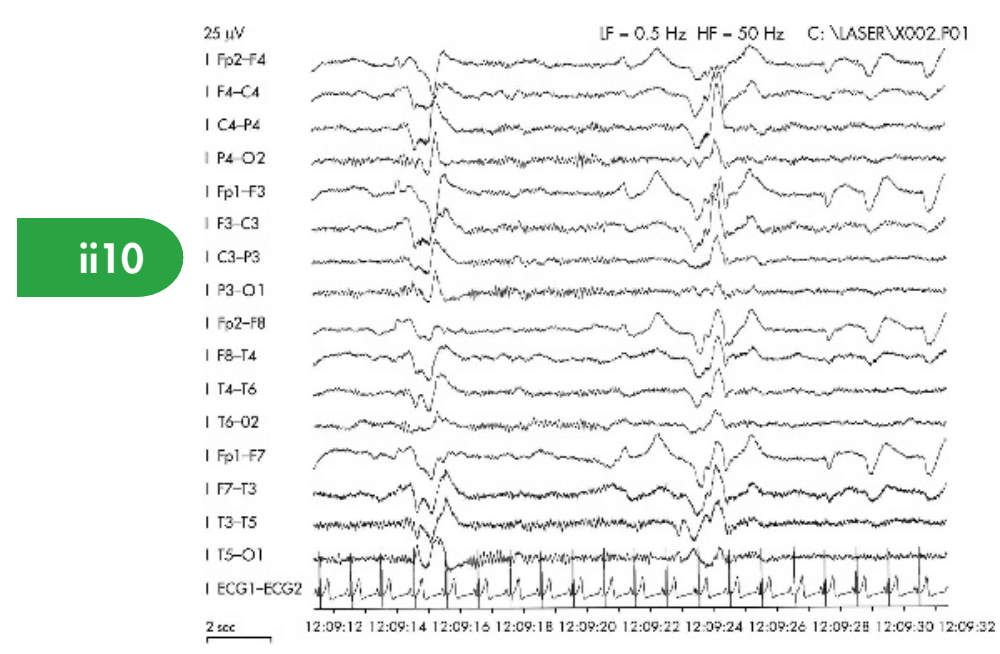

Figure 2 Stereotyped high amplitude periodic complexes in an adolescent female with subacute sclerosing pan-encephalitis.

neurological outcome. Unfortunately, predictive factors are difficult to identify from most studies, since these are small, retrospective, and with varying time intervals between symptomatic onset and EEG or treatment.

As with encephalopathies, EEG is most useful in acute encephalitis to identify seizures as a complicating factor.

\section{Subacute sclerosing pan-encephalitis (SSPE)}

This neurodegenerative complication of measles virus infection in childhood is worthy of mention, being one of very few disorders in which the EEG shows relatively specific or pathognomonic changes. Although rare, UK neurologists are likely to see more cases in years to come because of the decline in measles vaccination following the MMR/autism scare. The characteristic EEG picture is of stereotyped high voltage periodic complexes, usually generalised or bilateral (fig 2). Morphology of the complexes is highly stereotyped within an individual, but differs between patients. The periodicity varies from a few to many seconds, with a gradual reduction of the interval between complexes, and eventual disappearance of complexes as disease progresses. Background cerebral activity between complexes is normal initially, with increasing slow activity and then attenuation in later stages. The complexes are usually associated with myoclonic jerks (the myoclonus may be negative). Most SSPE presents in children and adolescents. Adult onset cases are rare, but clinical and electrographic features can be atypicalprominent early visual symptoms, lack of dementia, and a slow EEG without stereotyped complexes.

\section{HIV infection}

Non-localised or focal EEG abnormalities can occur, depending on the nature of opportunistic infection and other central nervous system involvement. In HIV encephalopathy, the usual finding is mild EEG slowing. Epileptiform discharges occur in association with seizures (up to $10 \%$ of patients with HIV dementia complex).

\section{DEMENTIA}

EEG would be a powerful tool if it was able to distinguish reliably between the worried well and those with minimal/ mild cognitive impairment, or predict which patients will go on to develop progressive cognitive decline. In practice, EEG is usually normal until there is little clinical doubt about the likelihood of dementia. It is uncertain yet whether quantitative EEG methodologies, which identify abnormalities in frequency or spatial distribution of cerebral rhythms not clearly visible to the eye, or longitudinal EEG studies in an individual patient, will be more diagnostically helpful especially in early detection of dementias.

There is limited change in the EEG in the normal aging brain. After the age of 85 years, $\alpha$ rhythm frequency declines slightly to around 7-8 Hz. Isolated or intermittent temporal slow waves may be seen in up to one third of healthy subjects over the age of 65 years. Their basis and whether they have any pathological significance is uncertain, but vascular factors are probable.

Interpretation of studies which have evaluated EEG in various neurodegenerative diseases is compounded by inaccuracy of purely clinical diagnosis and limited neuropathological data, inclusion of cases with varying stages of disease, inadequate control groups, and sometimes unclear description of EEG findings. Nevertheless, some general comments can be made about the range of EEG changes that are expected in more common dementias.

\section{Alzheimer's disease}

In the early stage, with mild cognitive impairment, the EEG is usually normal. As disease progresses, $\alpha$ rhythm slows in frequency and then disappears, and in the moderately or severely demented patient, the EEG is dominated by slow activity. The abnormalities are usually diffuse, but may sometimes show emphasis or be most pronounced over frontal or temporal lobes. A few patients show periodic sharp waves or epileptiform discharges, which may be associated with myoclonic jerks or seizures, but if prominent and there are atypical clinical features, other diagnoses should be considered. A number of small case series have reported patients in which apparently progressive memory dysfunction presenting as dementia is due to unrecognised complex partial seizures. These patients show epileptiform discharges over temporal lobes, and introduction of antiepileptic drug treatment leads to improvement in cognitive deficit. Pointers to such cases include a previous history of epilepsy, fluctuation in memory function, and absence of progressive deterioration on repeated psychometric testing. It may be necessary in some cases to monitor interictal EEG for up to 24 hours to identify epileptiform discharges occurring during nocturnal sleep only.

\section{Vascular dementia}

$\alpha$ rhythm may be preserved for longer than in Alzheimer's disease, or there may be prominent intermittent temporal slow activity.

\section{Lewy body dementia}

Some cross sectional studies have reported a greater degree of EEG slowing, and more focal slow activity in temporal regions in Lewy body dementia compared with Alzheimer's disease.

\section{Fronto-temporal lobar dementia (FTLD)}

It is usually said that the EEG is normal in FTLD. However, reappraisal of EEG findings with the benefit of pathological confirmation of diagnosis in larger numbers of cases shows that slowing occurs in up to $60 \%$, with correlation between degree of EEG abnormality and dementia. Abnormalities are 
most pronounced in patients with temporal lobe variant FTLD. Hence, EEG is not a reliable discriminator between FTLD and Alzheimer's disease either in the early or late stages of these disorders.

\section{Pseudodementia}

Due to psychiatric illness, the EEG is normal.

\section{Creutzfeldt-Jacob disease (CJD) and other prion disorders}

Periodic complexes (PC) are a sufficiently characteristic EEG finding in sporadic CJD (SCJD) for this pattern to be included by the World Health Organization as one of the criteria for diagnosis. The complexes usually have a bilateral or generalised distribution, and repetition rate of around $\mathrm{l} \mathrm{Hz}$ (ranging from $0.5-2 \mathrm{~Hz}$ ). Myoclonic jerks are usually present when PC develop, and may or may not be time locked to the complexes. Variations do occur-focal or lateralised complexes have been reported (such as occipital PC in the Heidenhain variant), and morphology of the complexes overlaps with that of triphasic waves and PEDs. Unsurprisingly, textbooks rarely provide a clear definition of what constitutes a periodic complex. The German CJD surveillance group has proposed objective EEG diagnostic criteria for purposes of epidemiological or multi-centre studies:

- strictly periodic cerebral potentials (most of duration 100$600 \mathrm{~ms}$ ) with intercomplex interval of 0.5-2 seconds

- generalised and lateralised complexes accepted

- at least five repetitive intervals to rule out semi-periodic activity.

About $65 \%$ of patients with SCJD will show PC in the EEG. However, in early stages of disease, the EEG may be relatively normal or non-specifically slow, and serial recordings should be performed every 3-4 weeks, if there is suspicion of CJD. Absence of PC in the EEG after 12 weeks duration of illness has been said to be a strong pointer against the diagnosis of CJD, unless of the atypical long duration form. In addition to $\mathrm{PC}$, the EEG shows progressive change in background cerebral rhythms, with loss of normal activity, increasing slow activity, and then decline in amplitude, eventually with

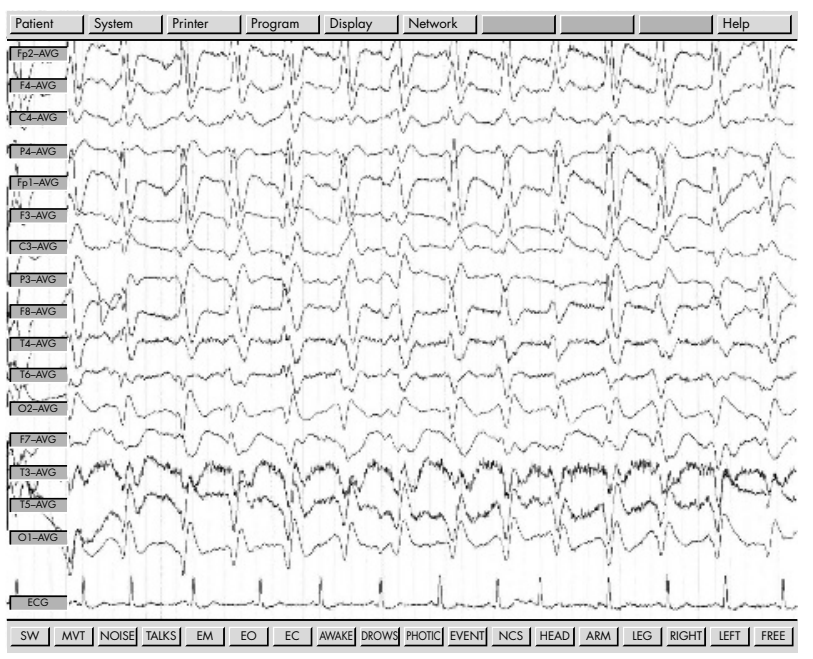

Figure 3 Characteristic EEG findings in sporadic Creutzfeldt-Jacob disease (sCJD): periodic complexes at approximately 1 per second, and very low amplitude featureless background between complexes. a featureless appearance between complexes (fig 3). PC usually disappear in the terminal stage of disease.

The reason why PC are not seen in a significant minority of patients with SCJD is unclear. The occurrence of periodicity may depend on how diffusely or extensively disease affects cortex, which in turn may be influenced by molecular and genetic factors. PC in the EEG are strongly correlated with methionine homozygosity or heterozygosity at codon 129 of the prion protein gene, and with type 2 pathological prion protein.

Although diagnostic specificity of PC for SCJD is high (around 90-95\%), PC are not pathognomonic of sCJD-they are described in other dementias (Alzheimer's disease, Lewy body, vascular) and toxic encephalopathies such as those caused by lithium. However, the clinical context is paramount. In a recent necropsy based study, combination of a typical EEG and typical clinical findings gave a positive predictive value of $99 \%$ for sCJD. Hopefully, in time, clinical and EEG criteria together with other data (14-3-3 protein in CSF and MRI) will provide a secure ante-mortem diagnosis of SCJD without the need for brain biopsy.

The presence of PC helps to rule out other prion disorders or transmissible spongiform encephalopathies. PC are not found in kuru, familial fatal insomnia, Gerstmann-StausslerScheinker syndrome (with the exception of very rare cases) or in variant CJD.

The overlap between triphasic waves, periodic epileptiform discharges and periodic complexes The morphology and other EEG characteristics of these different phenomena overlap. Fluctuation may give the appearance or impression of seizure activity, and PC can be abolished or attenuated by diazepam. Thus, even experienced electroencephalographers may have difficulty in distinguishing non-convulsive status epilepticus from an acute/subacute toxic or metabolic encephalopathy, or a rapidly progressive dementia. As always, EEG interpretation must be in clinical context, and in conjunction with other laboratory data. A few cases of SCJD presenting as complex partial status have been reported, with variable clinical and EEG response to seizure suppressing treatment. It is uncertain whether these patients really do have status epilepticus as part of the spongiform encephalopathy. More probably, misdiagnosis occurs because the clinical course, decline in conscious level, and development of PC in the EEG are particularly rapid.

Another problematic area is the significance of periodic epileptiform discharges (PEDs) with respect to clinical or subclinical seizures. PEDs occur in acute processes-strokes, tumours, and cerebral infections-that are themselves associated with epileptic seizures, impaired conscious level, and neurological dysfunction. PEDs are strongly correlated with refractory seizures (particularly focal motor, EPC, secondary generalised), and can appear during the course of convulsive and non-convulsive status epilepticus. However, not all patients with PEDs have seizures. How then to decide whether antiepileptic treatment is required in a patient with neurological dysfunction or impaired conscious level whose EEG shows PEDs? Clearly, treatment is necessary if there are overt clinical seizures, and prudent if there is a previous history of epilepsy. Treatment should also be considered if neurological dysfunction or impaired conscious level fluctuates or is episodic over time periods of minutes or possibly hours, suggesting an ictal basis. Whether EEG features suggesting electrographic seizure activity 
necessitate treatment is less certain. Identification of ictal EEG changes can be difficult, even when using specific criteria such as rhythmicity, frequency, and evolution of discharges or periodic features, and such criteria are not used consistently. Moreover, many district general hospitals in the UK do not have ready access to EEG equipment or trained personnel. Service availability of continuous EEG monitoring is even more limited. Routine EEG will not be adequate in certain patients: both coma and presence of PEDs have been shown to predict delayed time ( $>24$ hours) to first seizure in patients with seizures detected by continuous EEG. Some authorities in the USA advocate aggressive antiepileptic treatment in comatose patients with repetitive electrographic seizures or continuous spikes/periodic discharges, even when there are no clinical signs of seizures. There is, however, no good evidence from appropriately conducted clinical trials for improved outcome in such cases.

\section{AMNESIC STATES}

Patients with transient global amnesia have normal EEGs, unless the recording is done fortuitously during the acute event, when temporal slow activity may be seen. EEG referral should be reserved for those cases in which there is high clinical suspicion or likelihood of transient epileptic amnesia-in which the amnestic episodes are recurrent and brief, or of very long duration (complex partial status epilepticus lasting days).

\section{THE EEG IN PSYCHIATRIC DISORDERS}

A wide range of EEG abnormalities, often subtle and bordering on normal variants, has been described in psychiatric conditions, including personality disorders. None appears to be of diagnostic or prognostic value. Overall, there is low EEG yield in patients presenting with purely psychiatric symptoms. This is especially so in young subjects with new onset psychotic disorders featuring auditory and visual hallucinations. Such hallucinations in isolation of any other clinical pointers are hardly ever indicative of epilepsy. Even in neurological conditions that manifest initially with psychiatric features-for example, variant CJD-EEG is usually normal in the early stages. In middle aged or elderly patients with new onset psychiatric illness, EEG can be more helpful in detection of organic brain syndromes, with diffuse abnormalities indicating a neurodegenerative or encephalopathic process, or focal slow activity alerting to a space occupying lesion.

EEG is essential for identification of de novo absence status of late onset. This may occur without a preceding history of epilepsy, and be triggered by abrupt withdrawal of benzodiazepine or psychotropic drugs. Some cases are associated with metabolic disturbance or alcohol abuse. A high index of suspicion is required, as clinical manifestations are very variable. They range from a mild acute confusional state with mental dullness, irritability, patchy amnesia, and slight motor retardation to profound stupor with catatonia and loss of sphincter control. Primary misdiagnosis of a psychiatric disorder is very common. The EEG confirms the organic basis of the confusional state, and typically shows continuous or repetitive generalised/diffuse spike wave discharge (1-4 Hz). A characteristic of the condition is rapid clinical and EEG resolution with intravenous benzodiazepine (diazepam or lorazepam). Patients with de novo absence status and no prior history of epilepsy do not usually require long term antiepileptic drug treatment.

\section{REFERENCES}

1 Ebersole JS, Pedley TA, eds. Current practice of clinical electroencephalography, 3rd ed. Lippincott Williams and Wilkins, 2003.

- Authoritative multi-author reference text, covering the cellular basis of EEG, technological aspects, normal EEG across all age groups, and comprehensive description of clinical applications of EEG. Also includes chapters on evoked potentials and intra-operative monitoring.

2 Blume W, Kaibara M. Atlas of adult encephalography, Lippincott-Raven, 1995.

3 Goldenshohn ES, Legatt AD, Koszer S, et al. Goldenshohn's EEG interpretation: problems of overreading and underreading, 2nd ed. Futura Publishing Co Inc, 1999

- Practical guides to interpretation of EEG; useful conjunctive to standard EEG texts. Both atlases include a full range of normal and abnormal EEGs (including atypical records/variants) with detailed accompanying legends and summaries of key points. The Goldenshohn's atlas particularly stresses the piffalls of EEG evaluation and the problems that can result from over- and under-interpretation.

4 American Clinical Neurophysiology Society. Journal of Clinical Neurophysiology: official publication of the American Clinical Neurophysiology Society with issues devoted to specific topics and including original research papers.

- Volume 21 (5) 2004 covers clinical neurophysiology in critical care and diagnosis of encephalopathy, and includes appraisal of continuous EEG monitoring in detection of seizures and ischaemia, and the role of neurophysiological assessment to evaluate prognosis in ICU patients.

5 Hogh P, Smith SJ, Scahill RI, et al. Epilepsy presenting as AD: electroclinical features, and response to treatment. Neurology 2000;58:298-301.

- A reversible/treatable pseudodementia

6 Chan D, Walters RJ, Sampson EL, et al. EEG abnormalities in fronto-temporal lobar degeneration. Neurology 2004;62:1628-30.

- Reappraisal of EEG findings in this increasingly recognised dementia.

7 Steinhoff BJ, Zerr I, Glatting $M$, et al. Diagnostic value of periodic complexes in Creutzfeld-Jakob disease. Ann Neurol 2004;56:702-8.

8 Zerr I, Schulz-Schaeffer WJ, Giese A, et al. Current clinical diagnosis in Creutzfeldt-Jakob disease: identification of uncommon variants. Ann Neurol 2000;48:323-9.

- The German CJD group has published extensively on the value of commonly used clinical tests including EEG in diagnosis of CJD. 